Sessions d'étude - Société canadienne d'histoire de l'Église catholique

\title{
L’évolution du Séminaire de Chicoutimi
}

\section{Jean-Paul Laliberté}

Volume 40, 1973

URI : https://id.erudit.org/iderudit/1007252ar

DOI : https://doi.org/10.7202/1007252ar

Aller au sommaire du numéro

Éditeur(s)

Les Éditions Historia Ecclesiæ Catholicæ Canadensis Inc.

ISSN

0318-6172 (imprimé)

1927-7067 (numérique)

Découvrir la revue

Citer cet article

Laliberté, J.-P. (1973). L'évolution du Séminaire de Chicoutimi. Sessions d'étude -

Société canadienne d'histoire de l'Église catholique, 40, 131-134.

https://doi.org/10.7202/1007252ar

Tous droits réservés @ Les Éditions Historia Ecclesiæ Catholicæ Canadensis Inc., 1974
Ce document est protégé par la loi sur le droit d'auteur. L'utilisation des services d’Érudit (y compris la reproduction) est assujettie à sa politique d'utilisation que vous pouvez consulter en ligne.

https://apropos.erudit.org/fr/usagers/politique-dutilisation/ 


\section{L'évolution du Séminaire de Chicoutimi}

Je dirai à bâtons rompus ce que j'ai vécu, reliant certaines choses à ce que $\mathrm{M}^{\mathrm{gr}}$ Simard a énoncé. J'ai connu, comme élève, une partie de la période tranquille de l'histoire du Séminaire et $\mathbf{M}^{\mathrm{gr}}$ Simard aux divers postes qu'il a occupés. Par la suite, j'ai eu l'occasion de travailler avec $\mathbf{M}^{\mathrm{gr}}$ Alphonse-Elzéar Tremblay comme collaborateur immédiat, puisqu'il était supérieur du Séminaire alors que j'étais directeur des élèves pendant six ans; je faisais partie du Conseil de la maison et j'ai pu suivre d'assez près l'évolution de ce temps-là.

$\mathrm{M}^{\mathrm{gr}}$ Simard a rappelé la fondation des sections classiques dans certaines écoles secondaires et il a dit un mot de la Fédération des Collèges classiques. Ces sections ont continué d'opérer et de progresser sous le supériorat de $\mathrm{M}^{\mathrm{gr}}$ Tremblay. Puis, pour diverses raisons, elles ont été abandonnées. Certaines furent le noyau de fondation de nouveaux collèges, comme ce fut le cas à Saint-Joseph d'Alma pour le collège Champagnat; d'autres, comme celles de La Malbaie ou de Baie-Saint-Paul, ne faisaient plus partie du diocèse, Charlevoix ayant été rattaché au diocèse de Québec.

$\mathrm{M}^{\mathrm{gr}}$ Tremblay a donné beaucoup de son temps à la Fédération des Collèges classiques. Et je pense que, pendant cette période, un grand pas a été fait lorsque fut établi un Secrétariat permanent et construit un Siège social. Il faut attribuer cette évolution à la nécessité de créer des liens plus étroits entre les différents collèges, et même entre les professeurs. C'est au cours de cette période que la question des subsides du gouvernement fédéral aux universités se posa. Les collèges classiques étaient alors affiliés aux diverses universités dont les Facultés des arts décernaient la baccalauréat, contrairement aux collèges de langue anglaise qui étaient assimilés à l'enseignement universitaire. Ces derniers profitaient des subventions fédérales tandis que les octrois destinés aux collèges classiques de langue française étaient retenus par les universités auxquelles les collèges étaient affiliés sans vivre dans la même institution. Cette situation désavantageuse contribua largement à unir les forces en vue de former un front commun et de récupérer les sommes dues aux collèges classiques. C'est là que le Siège social devenait nécessaire. On organisa donc une souscription, et je me permets de dire que le Séminaire de Chicoutini a été l'un des donateurs les plus généreux, ayant souscrit plus de $\$ 25,000$. 
Pendant le supériorat de $\mathbf{M}^{\mathrm{gr}}$ Tremblay, il faut aussi signaler la séparation bien distincte entre le niveau proprement collégial, Philo I et Philo II, et les classes de grammaire et de lettres. De là la formation de ce qu'on appelait "philosophat ". Cette double structure a fonctionné pendant un certain temps, avec des directions et des communautés distinctes.

C'était l'époque de la parution du Rapport Parent; les tranches en étaient publiées successivement. C'était aussi l'époque de l'établissement du ministère de l'Éducation du Québec. Pendant ces années, alors que $\mathbf{M}^{\mathrm{gr}}$ Tremblay était supérieur, il y a eu incitation au développement de la part du ministère de l'Éducation, par l'intermédiaire de M. Jean-Marie Martin qui y détenait un poste important. A ce moment, ont été décidés les constructions ou agrandissements de Jonquière et de Chicoutimi. Je le mentionne à cause des répercussions dont l'influence fut considérable. Le Séminaire de Chicoutimi était résolu, en principe du moins, à effectuer des agrandissements et une modernisation de ses immeubles, sans avoir jamais songé, cependant, à faire des transformations et des constructions de l'envergure de celles qui ont été réalisées. C'est à l'instigation du ministère de l'Éducation que le projet a pris énormément d'ampleur puisqu'on jumela les projets de Jonquière et de Chicoutimi. A ce moment, si je me souviens bien, la première tranche du Rapport Parent était déjà publiée, et on entrevoyait, paraît-il, un développement dans la région du Saguenay, et personne n'ignore l'esprit de rivalité qui existait entre Jonquière et Chicoutimi. Chicoutimi qui avait toujours dominé dans le domaine de l'éducation ne pouvait pas se permettre de laisser diminuer son influence. Si Chicoutimi ne participait pas à un projet avec Jonquière, Jonquière se développerait seul comme centre d'éducation collégiale.

Et Chicoutimi conserva bien la première place. Le Séminaire s'agrandit; c'est l'ingénieur Lamarre, de Jonquière, qui en dressa les plans. On entreprit la construction d'un centre de services: laboratoires, salles de classe, salles de cours et bibliothèque centrale. Et comme, selon les plans, la bibliothèque a été placée là où se trouvait la chapelle et l'auditorium, ce dernier a été déménagé dans une nouvelle construction. On construisit aussi deux résidences d'étudiants. C'était pendant la période d'évolution ou de révolution. Le petit séminaire diocésain ne savait trop où l'amenait cette révolution. L'avenir des petits séminaires diocésains était incertain et on ne savait comment serait assuré le recrutement sacerdotal. Afin de pourvoir à toute éventualité, on affecta une des résidences à la formation des futurs ecclésiastiques alors que la deuxième recevait les étudiants qui se destinaient aux autres professions. Tous les étudiants suivaient les mêmes cours, cependant. 
Il va sans dire que la construction ne se fit pas sans difficulté. C'est à ce moment que je suis arrivé au Séminaire et j'étais supérieur quand la construction s'est terminée. Le coût dépassait les possibilités financières du Séminaire et, par surcroît, on dut changer de constructeur au cours des travaux. Pour faire face à la situation, le Séminaire songea à émettre des obligations. Mais, à la dernière minute, des hommes de loi découvrirent dans la charte du Séminaire, charte qui datait d'avant 1900 mais qui avait été créée par une loi, un article mentionnant que toutes les propriétés du Séminaire de Chicoutimi (propriétés qui avaient été cédées par l'évêché du temps ou par d'autres) devaient demeurer entre les mains d'ecclésiastiques romains pour fins d'éducation. La découverte de cet article mettait fin à l'espoir d'èmettre des obligations. Entre-temps, cependant, un prêt d'importance dépassant le million, avait été consenti par une banque. Celle-ci avait consenti le prêt en comptant sur l'émission d'obligations, ce qui amena des problèmes par la suite. Mais cette erreur a largement servi les intérêts du Séminaire puisque le prêt a permis de compléter la construction qui s'est terminée à l'automne 1965.

A la fin du supériorat de $M^{\text {gr }}$ Tremblay, une collaboration s'établit avec le Collège du Bon-Pasteur. Le Séminaire a ouvert certains de ses cours aux jeunes filles, particulièrement les cours de sciences. En retour, des élèves du Séminaire allaient prendre des cours au Collège du BonPasteur.

Le Séminaire s'ouvrit davantage encore à l'automne 1965, quand il occupa ses nouveaux locaux. Il en loua une partie à l'École normale Cardinal-Bégin (école normale d'hommes) qui existait à Arvida jusqu'alors. L'année suivante, il y eut fusion de trois écoles normales: Cardinal-Bégin, Bon-Pasteur et Bon-Conseil. Ces écoles fusionnées continuèrent leur enseignement dans les locaux du Séminaire et dans ceux du Bon-Conseil.

Le Rapport Parent continuait à paraître, et pointait à l'horizon l'idée de fondation de ce qu'on appelait alors les instituts. Le premier comité en vue de la fondation des instituts a été mis sur pied en novembre 1964. Il y avait dans la ville de Chicoutimi treize institutions qui donnaient au moins une année de collégial. Plusieurs de ces institutions sont disparues par la suite, de sorte qu'en 1967 il n'en restait que sept, je crois. D'aucuns envisageaient la mise sur pied d'un institut à Chicoutimi pour septembre 1965, mais le Frère Desbiens et le sous-ministre Bergeron ont refroidi l'enthousiasme de plusieurs à l'occasion de certaines rencontres, et les Iravaux ont continué dans cette optique, mais plus lentement. C'est à ce moment que je suis arrivé, en 1965, comme supérieur du Séminaire, ainsi 
que je l'ai mentionné en parlant de la construction. Le travail a continué de s'opérer en vue de l'établissement d'un collège d'enseignement général et professionnel, qui est devenu, comme on le devine, le nom des instituts projetés, et c'est le 14 juillet 1967 que le collège de Chicoutimi a été fondé. A ce moment, le CEGEP, assumant la responsabilité de l'enseignement collégial, établit la majorité de ses services dans les immeubles du Séminaire de Chicoutimi, qui retint, cependant, l'une des deux résidences. Ce sont ces événements qui firent qu'en 1967 le Séminaire de Chicoutimi a obtenu une nouvelle charte.

Jean-Paul Laliberté, V.F., L. ès Sc., Chanoine honoraire,

Curé de la Paroisse Saint-Joseph d'Alma, Supérieur du Séminaire 1965.1967. 